\title{
Incidencia del merchandising en las empresas de accesorios de lujo para autos de alta gama en la ciudad de Barranquilla. Caso: tunning imports
}

Incidence of merchandising in the companies of luxury accessories for high-range cars in the city of Barranquilla. Case: tunnnig imports.

DOI: 10.21803/adgnosis.v7i7.301

\section{Resumen}

Se pretenden evaluar las variables de Merchandising que intervienen en la toma de decisiones de los compradores de las empresas del sector accesorios, y su influencia en la productividad, qué de manera directa, incide en el crecimiento de estas empresas comerciales en la Región Caribe Colombiana, en especial en la ciudad de Barranquilla, así el presente artículo toma como estudio de caso la empresa Tunning Imports. Para esta evaluación se hace uso de herramientas investigativas y documentales, a través de la revisión de 16 referencias, cuyos resultados mostraron una gran influencia en el comportamiento del consumidor establecido y en la toma de decisiones de este target que han llevado a un alto crecimiento de estas empresas en la ciudad, así como también un sondeo a través de encuestas al azar de clientes que se acercaron a la empresa.

Palabras clave: Merchandising, Arquitectura de la Tienda, Artículos, Comportamiento de los Consumidores, Estrategias.

\section{Abstract}

It is intended to evaluate the Merchandising variables that intervene in the decision making of the buyers of these important companies, and their influence on productivity, which, directly, affects the growth of these industries in the city of Barranquilla, and in Special in the north coast of Colombia, this article is based on the company Tunning imports, located in the city of Barranquilla. For this evaluation, investigative and documentary tools are used, through the review of 16 references, whose results showed a great influence in the behavior of the established consumer and in the decision making of this target that have led to a high growth of These companies in the city, as well as a survey through random surveys of customers who approached the company.

Keywords: Competitiveness, Government and institutions, Economic Development, Human Capital, Business Efficiency, Productive Infrastructure.

\section{Rubén Cubillos Coll 1 \\ rubenjr.cubillos@gmail.com}

\section{Diana Lastre Gómez²}

dianalastreg@gmail.com

Juan José Sarué Montalvo ${ }^{3}$

jsarue87@hotmail.com

Jonathan Sarué Montalvo ${ }^{4}$

jjsarue@gmail.com

\section{Cómo citar este artículo:}

Cubillos, R., Lastre, D., Sarué, J. \& Sarué, J. (2018). Incidencia del merchandising en las empresas de accesorios de lujo para autos de alta gama en la ciudad de Barranquilla. Caso: tunning imports- 7(7), p. 129148. DOI: 10.21803/adgnosis.v7i7.301

1. Administrador de Empresas, Universidad Autónoma del Caribe. MBA, Universidad del Norte. Investigador Grupo GEMS. Correo: rubenjr.cubillos@gmail.com 2. Psicologa. Licenciada en Piscología en Infantil. Maestría en Educación, Universidad del Norte. Correo: dianalastreg@gmail.com

3. Relaciones Internacionales, Universidad del Norte. Especialista en Mercadeo. Magíster en Mercadeo, Universidad Autónoma del Caribe. Correo: jsarue87@hotmail.com 4. Administrador de Empresas, Ceipa. Correo: jjsarue@gmail.com 


\section{Merchandising}

Para los entendidos, mercadólogos, y para los profesionales del marketing, el merchandising es el conjunto de técnicas y conceptos, cuyo propósito es incentivar la compra en el punto de venta, sin la intervención de vendedor alguno, haciendo uso de la ubicación, exhibición, colocación, administración del surtido (stocks) de mercancías y manejo de diversos elementos motivacionales en el punto de venta.

El merchandising tiene el propósito de maximizar la rentabilidad de cada metro cuadrado o lineal del establecimiento comercial, teniendo en cuenta que la rentabilidad es el resultado de multiplicar el margen individual de cada producto por el volumen de ventas, lo cual implica maximizar el volumen de ventas, administrando tanto el nivel de inventarios como el uso del espacio de exhibición (Lerma, 2003).

El término de la lengua inglesa es el resultado de unir el sustantivo "merchandise", que significa "mercancía" y el radical "ing", que expresa la acción, es decir, la acción mediante la cual se pone el producto en poder del consumidor, por ello se puede definir el "merchandising" diciendo que: Es el Marketing en el punto de venta, también conocido como "El Vendedor Silencioso / Micromercadotécnia".

"La parte del marketing que engloba las técnicas comerciales que permiten presentar al posible comprador final del producto o servicio en las mejores condiciones materiales y psicológicas. El merchandising tiende a sustituir la presentación pasiva del producto o servicio, por una presentación activa, apelando a todo lo que puede hacerlo más atractivo: colocación, fraccionamiento, envase y presentación, exhibición, instalación, etc." Academia Francesa de Ciencias Comerciales.

"Conjunto de estudios y técnicas de aplicación puestos en práctica, de forma separada o conjunta, por distribuidores y fabricantes con mi- ras a acrecentar la rentabilidad del punto de venta y la introducción de los productos, mediante una aportación permanente del producto a las necesidades del mercado y mediante la, presentación apropiada de las mercancías." Instituto de Merchandising de Chicago.

Por lo que implica tomar decisiones sobre:

- Selección del surtido.

- Niveles de stocks.

- Disposición interna del punto de venta.

- Presentación de los productos en el lineal.

- Diseño exterior del punto de venta.

- Ambientación del punto de venta.

- Gestión del surtido.

- Gestión del espacio del lineal de un punto de venta.

Básicamente, el merchandising se podría definir como el conjunto de técnicas que se aplican en el punto de venta, para así motivar el acto de compra de la manera más rentable, tanto para el fabricante como para el distribuidor, satisfaciendo, de esta forma, las necesidades del consumidor.

Así que se podría afirmar que el merchandising busca la optimización del manejo de productos escogiendo las ubicaciones adecuadas en función de variables, como podrían ser el lugar, el tiempo, la forma, la cantidad y la arquitectura interior del espacio.

Viendo esta definición del merchandising, está claro que es de vital importancia para el marketing, ya que son muchos los beneficios que esta técnica aporta desde el punto de vista estratégico. Entre estos beneficios, se encuentran los siguientes:

- Potenciación de la rotación de productos.

- Reducción del tiempo de compra.

- Cambio del concepto de "despachar" productos por "vender".

- Potenciar la rotación de productos. 
- Aprovechar al máximo el punto de venta.

- Sustituir la presencia "pasiva" por una presencia "activa".

- Potencias los productos "imán" del punto de venta.

- Crear una adecuada comunicación integral en el punto de venta

Desde el punto de vista del Merchandising, la rentabilidad es más importante para el Distribuidor que para el Fabricante; para esté, lo más relevante lo constituye el conjunto de acciones publicitarias y promocionales a nivel detallista, destinadas a atraer la atención de los clientes hacia su producto. En cambio, para el Distribuidor, lo más destacado es el conjunto de métodos que aseguran la rentabilidad óptima de la superficie de venta.

Todo ello puede resumirse en la siguiente frase, que expone explícita y coloquialmente el verdadero objetivo del merchandising, el cual no es más que "Colocar al producto en peligro de ser adquirido por el consumidor" (Cubillos \& Laverde, 2009).

Para este artículo de investigación, los autores han escogido la empresa Tunning imports, con el fin de definir cuáles son las estrategias de merchandising que más se ajustan a las necesidades de los clientes, ya sean prospectos o no para este tipo de empresas en la ciudad de BarranquillaColombia.

Tunning es una empresa dedicada a la importación, comercialización de autopartes, accesorios Tunning específicamente.

Debido a la alta demanda, actualmente por este tipo de accesorios se ha creado tunningimports.com para satisfacer a uno y cada uno de los clientes ya que es para ustedes y por ustedes que se esta y se sigue estando.

Más que una venta, nuestro enfoque son las personas; atenderlos, guiarlos, servirles en todo lo que se pueda de la mejor forma posible siempre haciendo la diferencia. Es por eso que constantemente se seguira innovando en nuestro servicio al cliente que es 24/7 (tunningimports.com, 2018).

Bien sabido es de todos, que los espacios comerciales cumplen una función específica de Vender: Para llevar a cabo dicho objetivo básico, el medio más usual es concentrar toda la fuerza atractiva en los escaparates, pues éstos son los espacios destinados a exhibir el género a la venta. Por ello, el emplazamiento de los aparadores debe ser entendido como la condición primordial a tener en cuenta, ya que cuando su ubicación es incorrecta no suele servir de mucho que los demás atributos del escaparate tengan un alto nivel de calidad. Otro aspecto ineludible en los proyectos de Vitrinismo es tener muy presente el concepto de visibilidad, puesto que cualquier producto necesita de una mínima perspectiva para poder ser apreciado y valorado.

Así, cuando se habla del lugar de ubicación de los escaparates de una tienda, siempre se suele remitir a la idea generalizada de unos espacios expositivos que suelen estar emplazados en la fachada exterior del edificio en que se aloja la tienda. Ahora bien, si se enfoca en un análisis más detallado del término y del mundo que le rodea, se notaran las diferencias sustanciales entre los escaparates de fachada, los interiores y los de pasillo, aunque todos ellos mantengan una relación de dependencia, a la vez que cumple igualmente con su función de reclamo (Cubillos \& Laverde, 2009).

El departamento de merchandising, suele estar organizado en líneas de mercancía con que cuenta una tienda, lo que permite la especialización del merchandising. Este departamento es responsable de comprar los bienes, de las relaciones con los proveedores, de la planeación del surtido y de trabajar con el departamento de promoción en la planeación d eventos especiales y campañas de publicidad. Más específicamente, 
este departamento implementa sus políticas de merchandising de la gerencia de la compañía mientras dirige a sus gerentes especializados en la formulación de una imagen consistente en la tienda a través de los productos que ofrecen al público, y tienen una relación más directa que los otros departamentos con las actividades de ventas que producen las ganancias, los cuales cumplen roles de soporte (Hasty \& Reardon, 1998).

\section{2.- Conceptos fundamentales de mer- chandising. (adaptado de Lerma, 2003)}

Atendiendo a lo anteriormente expuesto, el merchandising, tiene cuatro (4) grandes áreas básicas sobre las cuales se trabaja. En su orden son las siguientes:

A. Ubicación del almacén.

- Atracción

- Accesibilidad

- Urbanización

- Posibilidades futuras

- Tipo de establecimiento

- Tipo de clientela

- Tipo de zona.

- Superficie de venta
- Presentación vertical - elementos de la góndola.

- Número de estantes - presentación horizontal.

- Personalización e imagen corporativa.

C. Artículos

- El surtido

- Extensión y profundidad

- Artículos imán.

- Artículos gancho.

- Marcas, familias y categorías.

- Complementariedad.

- Rentabilidad.

- Colocación de los productos.

D. Motivación a la compra.

- Promoción

- Un producto

- Por marca

- Gama de productos

- Motivación en el punto de venta.

- Medios físicos

- Medios psicológicos

- Medios personales

- Estímulos visuales

\section{3.- Objetivos y beneficios del merchandising}

Objetivos del merchandising.

Macro Objetivos

El objetivo del merchandising es provocar mayor demanda del producto ya que ello significa mayor rotación, mayor utilidad, mayor eficiencia, mayor frecuencia de compra.

-Atraer la atención

-Crear el interés

-Despertar el deseo

-Accionar la compra

Nota: Esto corresponde a la tradicional formula: AIDA del marketing.

Objetivo General 
Es "Alcanzar la mayor rotación posible de los productos expuestos en el punto de venta".

\section{Objetivos Básicos}

- Organización: Optimizar la colocación de los productos para agilizar la lectura de la oferta.

- Gestión: Rentabilizar la rentabilidad de cada metro

- Seducción: Mejorar la atracción de los productos por su puesta en escena

\section{Objetivos Específicos.}

- Conseguir en cada uno de los anteriores objetivos al máximo el punto de venta, la gestión del merchandising pasa por alcanzar una serie de objetivos:

- Mostrar más atractivo el producto para el cliente mediante una adecuada exposición del mismo.

- Incrementar la afluencia de público al establecimiento, a través del tratamiento de los elementos exteriores como: vitrina, fachada y rótulos, que lo hagan más atractivo y sirvan para la recordación.

- Crear ambiente agradable y de animación para el cliente, para que comprar no resulte rutinario.

- Multiplicar los efectos de una campaña publicitaria o promocional. En general, el consumidor acude al establecimiento con un recuerdo leve del mensaje publicitario, es necesario que éste lo encuentre en las mejores condiciones en cuanto a su presentación, información, identificación y ubicación para que se traduzca en un efecto multiplicador de la campaña.

- Poner el producto en manos del consumidor de forma que se venda a sí mismo.
- Gestionar adecuadamente la superficie de ventas para que resulte rentable y atractiva al mismo tiempo. El tamaño de la superficie de ventas y el espacio del mismo asignado a cada producto son aspectos determinantes. Esta decisión estará en función del tiempo de exposición, de la naturaleza de los productos, de los tipos de implantación de los diferentes niveles de exposición, de los tipos de compra y de la publicidad en el punto de venta. Por otra parte, a cada producto se le debe asignar una parte del área de venta que no resulte ni excesiva ni insuficiente. Para la asignación del área de exposición de venta es preciso conocer la probabilidad de venta de cada producto.

- Impulsar la relación productor-consumidor a través de diferentes acciones conjuntas con el fabricante, tales como promociones, ofertas especiales, mejora de la gestión del área expositiva y determinación del surtido adecuado.

- Incrementar la rotación del producto.

- Atraer la atención del comprador hacia productos concretos a través de su ubicación estratégica en la superficie de ventas.

- Eliminar el stock de artículos poco vendibles, mediante el apoyo de ofertas o promociones.

- Incentivar a los clientes a Recorrer la tienda para comprar mayor cantidad de mercadería de lo que habían planificado.

- Lograr un Balance entre el espacio destinado a la movilidad del cliente y el espacio de vena.

\section{Beneficios del merchandising}

- Lograr seducir al cliente fortaleciendo la presentación. 
- Aumentar la rotación de los productos con ventas cruzadas.

- Incrementar la rentabilidad de la empresa a través de una óptima administración de los recursos

\section{Actuaciones centrales del merchandising}

- Diseño del embalaje, - o acorde con la naturaleza del producto- de los productos para hacerlos para hacerlos más atractivos y persuasivos.

- Lograr el relacionamiento y colaboración con los fabricantes.

- Tramitar adecuadamente el surtido para satisfacer a los clientes claves.

- Diseño de exhibiciones y de la publicidad en el punto de venta.

- Encargarse estratégicamente el punto de venta.

- Controlar y evaluar la óptima exhibición de los productos en el punto de venta.

- Incrementar la rotación de los productos y la rentabilidad del punto de venta.

- Generar buenas relaciones y apoyo con los distribuidores.

- Crear un ambiente propicio para generar ventas por impulso.

- Diseño de la arquitectura externa e interna del punto de venta

\section{Normativa del merchandising}

El merchandising, también implica, la aplicación de las siguientes normas básicas.
1. El producto adecuado, que corresponde con el surtido adecuado. Es, fundamentalmente, en el merchandising una buena selección de la calidad y el número de los productos.

2. La cantidad adecuada, hace referencia a la imperiosa necesidad de una buena gestión de stocks.

3. El precio adecuado, indica que hay que buscar el precio idóneo teniendo en cuenta factores como rentabilidad, coherencia, clientela, etc.

4. El momento adecuado, conlleva tener en cuenta la elección del momento en que se ofrece el producto (en el caso de productos estacionales) y, también, el momento en el que normalmente se decide la compra de un producto (paraguas cuando llueve, viajes antes de las vacaciones, etc.).

5. El lugar adecuado, está estrechamente relacionado con la implantación del producto en el punto de venta. Siendo esta una de las funciones principales del merchandising.

6. La adecuada forma de presentación y venta de los productos.

Tipos de merchandising.

- Merchandising permanente, es aquel que se realiza de forma continua a lo largo del año. Encargado de:

- La fidelización y captación de clientes.

- Genera vínculos establecimiento - cliente (el cual reconoce y se identifica con el punto de venta).

- El ambiente agradable (iluminación, temperatura, música...), la disposición interna establecimiento (entrada y recorridos), la distribución del espacio (puntos fríos-calientes), la gestión del área 
expositiva (tipo de surtido, tipos presentación, criterios colocación, gestión por categorías).

- Merchandising promocional, se aplica con el lanzamiento de un nuevo producto, oferta, regalo, etc. Aporta:

- Mayor agresividad comercial.

- Dinamismo y ayuda a combatir la estacionalidad de las ventas y productos (permitiendo incrementar nuestros beneficios).

- Cartelería de señalización, Material PLV (el tercer vendedor), Animación punto venta (Eventos), Vitrinas y exposiciones especiales (interiores / exteriores).

- Merchandising Estacional: Es el que se realiza en virtud de la relación de los productos con la época del año.

\section{Clases de Merchandising.}

El concepto de merchandising y las técnicas correspondientes, no siempre se utilizan de idéntica forma, porque los objetivos que se persiguen no siempre son los mismos.

Por ejemplo, si se tiene en cuenta el objetivo de máxima exposición de todo el establecimiento ante los clientes, ha de colocarse los productos de consumo frecuente, como la leche / pañales, lejos de la entrada y de las cajas de salida, es decir, en lugares que provoquen una ruta larga y obligada.

Ahora bien, si lo que se busca es el máximo volumen de venta de un producto, se colocara en un lugar de paso frecuente y máxima afluencia de público, o sea, en lo que se denominara como un punto caliente.

Si se quiere dar imagen de precios bajos se pondran carteles de ofertas en la fachada o al entrar. Por el contrario, si se desea transmitir una imagen de alta calidad, se pondra una fachada elegante, así el punto de venta llama a la elegancia. Todas las actividades de merchandising han de realizarse teniendo en cuenta los objetivos perseguidos por la empresa para conseguir así su máxima efectividad.

Pero no solamente los objetivos de la empresa exigen distintas clases de merchandising, sino también, las circunstancias de lugar, tiempo, etc., que rodean al establecimiento. Por ejemplo, no se emplea el mismo merchandising en época de rebajas que en temporada normal. Los productos se dispones de forma que puedan ser fácilmente tomados y comprados.

Por todo ello, se puede hablar de distintas clases de merchandising como resulta de los diferentes enfoques o puntos de vista desde done sea considerado. Ver la siguiente tabla:

Tabla 1.

Clases de merchandising

\begin{tabular}{|c|c|c|}
\hline 1 Según su naturaleza & b. & $\begin{array}{l}\text { Merchandising de presentación. } \\
\text { Merchandising de gestión. } \\
\text { Merchandising de seducción. }\end{array}$ \\
\hline $\begin{array}{l}\text { 2. Según la situación del } \\
\text { cliente }\end{array}$ & $\begin{array}{l}\text { a. } \\
\text { b. }\end{array}$ & $\begin{array}{l}\text { Para el cliente shopper. } \\
\text { Para el cliente buyer. }\end{array}$ \\
\hline 3. Según el ciclo de vida & $\begin{array}{l}\text { a. } \\
\text { b. } \\
\text { c. } \\
\text { d. }\end{array}$ & $\begin{array}{l}\text { De nacimiento. } \\
\text { De ataque. } \\
\text { De mantenimiento. } \\
\text { De defensa. }\end{array}$ \\
\hline 4. Según la orientación & D & $\begin{array}{l}\text { Merchandising técnico. } \\
\text { Merchandising comercial }\end{array}$ \\
\hline 5. Según quien realice & & $\begin{array}{l}\text { Merchandising de fabricante. } \\
\text { Merchandising de distribuidor. }\end{array}$ \\
\hline
\end{tabular}

Fente: Elaboración propia.

Nota: para efectos de este artículo, y acorde con la investigación, solo se tomaran los dos primeros de la clasificación.

\section{Según su Naturaleza}

\section{a. Merchandising de Presentación}

Es la manera de presentar los artículos y el punto de venta de modo que el acto de compra sea lo más fácil para el cliente y lo más rentable posible para el comerciante, tanto respecto al nú- 
mero de unidades vendidas como al margen de beneficio por unidad de producto.

A continuación, se enseñara el esquema del merchandising de presentación que también se puede denominar merchandising visual: todo lo que se ve, se vende; todo lo que se coge, se compra; es decir, lo que también se llama compra por impulso.

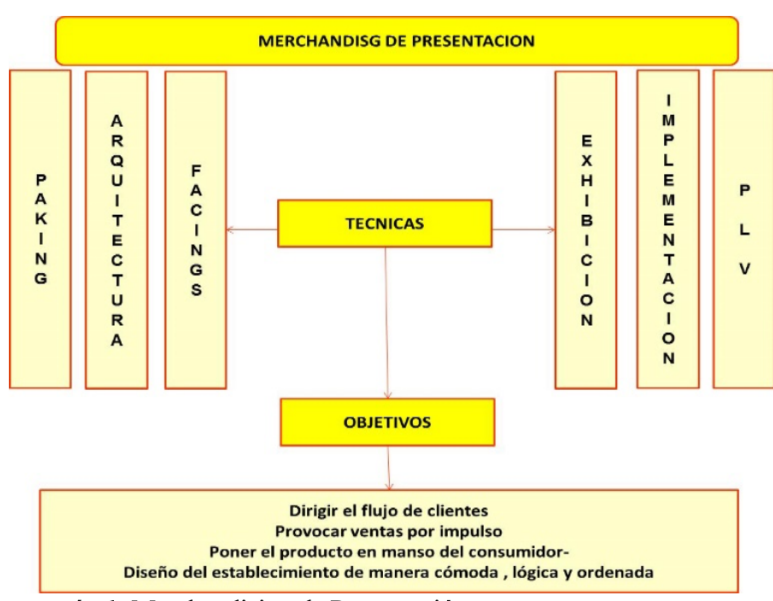

Ilustración 1: Merchandising de Presentación

Fuente: Libro Marketing para droguerías (Laverde, 2015).

El objetivo es "dirigir el flujo de clientes a las secciones o productos concretos, estimular las comprar por impulso y poner los productos al alcance del consumidor, ya que, a través de un diseño cómodo, lógico y ordenado del establecimiento, una presentación atractiva de los productos y aplicando técnicas que mejoren la presentación visual del producto se potencia su atractivo y en definitiva los hace más vendedores.

\section{Macro y Micro Visual merchandising.}

Cabe diferenciar el macro espacio de un establecimiento con el micro espacio de una zona en concreto del mismo.

En cada uno de ellos las técnicas a aplicar son diferentes. El Visual Merchandising puede ser interactivo con el cliente o el paciente. Una vitrina transmite los valores del punto de venta, un es- pacio dedicado a las consultas en un área privada con acceso al ordenador y a la impresión de folletos. Todo esto hay que saberlo transmitir al usuario o al potencial cliente.

\section{Macro Visual Merchandising}

Cuando se habla de macro, se piensa en el total del establecimiento.

- Es una sección del espacio en la que se transmite la experiencia de la marca a través de: ambiente iluminado de la manera adecuado, temperatura agradable, música tranquila, olor adecuado, discreción en la comunicación con el paciente, mostradores individuales que permiten que la gente no escuche a los demás, profesional adecuado y formado en todos los aspectos (desde saber coger una receta, mirar a los ojos al cliente, escuchar lo que dice, esperar a que el cliente se haya ido para irse y un largo etcétera que se da por sabido pero que cada vez se aplica menos).

- Sirve para crear una guía en el interior del local, por ejemplo, para hacer andar a los clientes en una dirección concreta.

- Permite una comunicación visual, se pueden usar gráficos, carteles colgando del techo para indicar zonas, flechas en el suelo, colores distintos para cada categoría o zona del punto de venta.

- Es una herramienta comercial.

- Es una oportunidad para dar a conocer actividades que se desarrollan dentro de la tienda: charlas formativas.

- Entretenimiento dentro del establecimiento. Un cliente mientras espera se le puede transmitir tranquilidad, confidencialidad, Se puede ofrecerle acceso a información a través de una serie de libros, revistas actualizadas.

- Forma de expresar la creatividad del equipo que trabaja en la tienda. 
Quizás hay un funcionario que pertenece a una banda de rock o un adjunto que toca el violín o un auxiliar que es del equipo de futbol y quieren dar a conocer esta actividad en su negocio. Pueden hacerlo a través del visual merchandising.

\section{Micro Visual Merchandising.}

En contraposición al MACRO, éste visual merchandising afecta a una zona más concreta del establecimiento y se puede diferenciar entre las distintas alturas de los niveles: altura alta, nivel de los ojos, nivel del suelo y extras.

- Nivel de altura alta se encuentra: gráficos, luz del establecimiento, arquitectura del local, posición de los productos, tratamiento de las paredes, color, señalética, vibración general del establecimiento.

- Altura a nivel de los ojos: posicionamiento de los productos, punto de venta, mostradores, displays encima del mostrador, puntos focales y puntos calientes.

- A nivel del suelo: rutas de circulación marcadas en el suelo, tipo de suelo, posicionamiento de productos, densidad.

- Extras: escaparates bien decorados, sensación que se lleva el consumidor que acude al establecimiento.

Entender al consumidor que acude a nuestro establecimiento.

Consumidor o cliente, es un término que puede genera cierta controversia.

Sin embargo, se pueden combinar ambos, unas veces vendrá alguien a adquirir un paquete de pañuelos y otras veces vendrá un adulto con un bebé a solicitar un producto específico para él bebe.

En un punto de venta, el uso de colores estri- dentes puede generar un impacto visual ganador, pero un exceso de visual merchandising pueden ser contraproducentes.

La idea de querer separar cada categoría de la tienda por colores, ha originado un espacio estridente, que no incita a la calma y si a la confusión.

Para algunos expertos esto es un mal ejemplo de visual merchandising en el diseño de interiores.

Cada audiencia responde diferente al uso de colores y materiales, por tanto, es muy importante conocer qué les gusta y qué no a la hora de diseñar un establecimiento que funcione en su efecto máximo.

También es necesario saber que los diseños se deben modificar cuantas veces hagan falta para encajar en el gusto de un mercado determinado.

El objetivo es diseñar un ambiente que anime a los usuarios a desarrollar una relación personal con el establecimiento.

Si uno es capaz de construir y operar un ambiente en el establecimiento que encaje con las necesidades particulares de los compradores o clientes, habrá conseguido el éxito del local.

El éxito del local no sólo depende del visual merchandising. Depende de la dirección, de saber transmitir esta misión y valores, estudiar qué objetivos son los que quiere conseguir, que el equipo trabaje de la manera adecuada.

El visual merchandising está intermedio de:

-Equipo de Marketing

-Creativos

-Actividad comercial.

Dando soporte a:

-Actividades promocionales, eventos del ca- 
lendario (día mundial de la diabetes), campañas de publicidad que hacen los laboratorios y que se tiene que conocer.

Debe potenciar la venta, inspirar la compra, hacer al producto atractivo.

¿Quiénes participan en este proceso?

- Clientes

- Elementos creativos

- Equipo que implementa el visual merchandising.

- Laboratorios

- Diseñadores del producto

- Compradores o Clientes

- Equipo de comunicación

- Diseñadores del establecimiento

- responsables del diseño del espacio.

- Equipo de Marketing.

Por tanto, el visual merchandising no es una disciplina sola, que, en definitiva, pretende guiar al consumidor e influir en su comportamiento a la hora de la compra apoyándose en los siguientes elementos:

- Un ambiente agradable.

- Una buena distribución y disposición, tanto del espacio como del establecimiento.

- Un surtido adecuado en cantidad, calidad y variedad.

Este tipo de merchandising se fundamenta en:

- El producto adecuado.

- La cantidad adecuada.

- El lugar adecuado.

- La forma adecuada.

Por ejemplo, los establecimientos de descuento, no ofrecen espectáculo alguno, presentan los productos de forma sencilla de fácil acceso, muy económicamente, dentro de las cajas o envase y sin intención de seducir a la compra, ya que sólo persiguen facilitar la compra y ahorrar costes.

\section{Merchandising de Gestión.}

Es la segunda etapa en la evolución del merchandising. Consiste, como su nombre indio, en gestionar el espacio para obtener el máximo rendimiento posible del lineal (espacio destinado por el establecimiento a la exposición y venta de los productos).

Trata de optimizar el lineal determinando su tamaño, las distintas familias, marcas y artículos que los forman, teniendo en cuenta la rotación de los productos, el rendimiento por metro cuadrado de la superficie de venta, el rendimiento por metro de lineal, etc.

El merchandising de gestión se basa en la recogida y análisis constante de información, principalmente generada en el propio establecimiento.

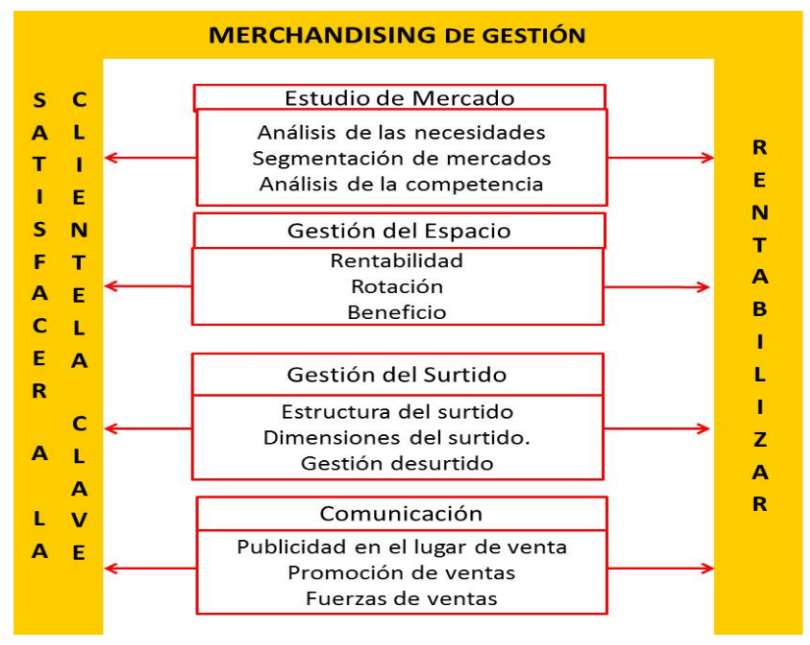

Ilustración 2. Merchandising de Gestión

Fuente: Libro Merchandising para Droguerías (Laverde, 2015).

El establecimiento tiene un gran poder de recomendación en todos los productos para cuya adquisición. Esto junto con la publicidad, ha hecho que existan unas marcas que gozan de gran popularidad y confianza entre los consumidores.

Al consumidor le gusta ver estas marcas expuestas en el punto de venta, ya que identifican 
ese lugar como próximo y dotan de prestigio al establecimiento.

Es responsabilidad del administrador saber dónde, cuándo y cómo colocar esas marcas y saber mezclar éstas con otras que aporten valor y beneficio. Para ello, es necesario que el lugar destinado a la venta disponga del mayor espacio posible. La gestión de este espacio de exposición busca su optimización económica.

\begin{tabular}{|l|l|}
\hline \multicolumn{1}{|c|}{ MERACCIÓN } & \multicolumn{1}{c|}{ CONECTAR } \\
\hline Medios físicos & $\begin{array}{l}\text { Punta de góndolas, islas, } \\
\text { exhibidores }\end{array}$ \\
\hline Medios psicológicos & $\begin{array}{l}\text { Promociones de ventas } \\
\text { Vendedor, Animador de } \\
\text { punto de venta, Stan de } \\
\text { demostración o } \\
\text { degustación. }\end{array}$ \\
\hline Medios personales & SEDUCCIóN \\
\hline Medios audiovisuales, \\
Creación de ambientes
\end{tabular}

Ilustración 3. Merchandising de Seducción

Fuente: Libro Merchandising para Droguerías (Laverde, 2015).

\section{Merchandising de Seducción o animación}

Es tal vez uno de los conceptos menos desarrollados en los puntos de venta y más audaces utilizados en la actualidad; sin embargo a nivel se supermercados se realizan actividades como degustaciones, promociones, colocaciones especiales, decoración, mobiliario iluminación, etc. con la utilización de técnicas físicas, como por ejemplo presentación de cestas y bandejas, islas completas de un producto, técnicas psicológicas como las ofertas, presentaciones múltiples que influyen mucho siendo cosas que le encantan al cliente y técnicas de espectáculo como lo son los juegos, concursos, presencia de personajes, demostraciones, etc.

De este tipo de merchandising se conocen bastante, ya que a diario en supermercados y centros comerciales se enfrentan a este tipo de actividades que los llevan a hacer parte del espectáculo y a vivir una buena experiencia que deja como resultado la compra del producto, donde finalmente la marca logra su objetivo.
Éste consiste en lograr que la compra se haga de manera entretenida, convirtiendo el punto de ventas en un área estimulando los sentidos del cliente; Vista, Oído Tacto, Olfato, Gusto. Todo este desarrollo se lleva bajo la premisa de la ecuación: ATRACCION + CONEXIÓN = SEDUCCION

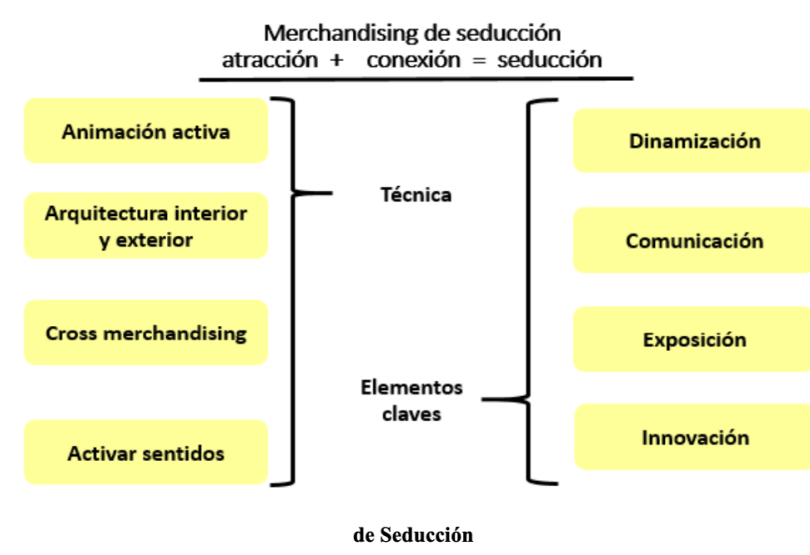

Ilustración 4. Ecuación de Merchandis

Fuente: Libro Merchandising para Droguerías (Laverde, 2015).

Siendo la atracción la máxima expresión de visibilidad, y la conexión una estrategia de comunicación dirigida al shopper, innovación tocando los otros sentidos, que al combinarlas con la utilización de técnicas físicas, como por ejemplo presentación de cestas y bandejas, islas completas de un producto, técnicas psicológicas como las ofertas, presentaciones múltiples que influyen mucho siendo cosas que le encantan al cliente y técnicas de espectáculo como lo son los juegos, concursos, presencia de personajes, demostraciones, etc.

De este tipo de Merchandising se han conocido poco, ya que a diario en supermercados y centros comerciales se enfrentan a un POP inerte y pasivo, sin embargo, hay empresas que se han atrevido a ser parte del espectáculo y a vivir una muy diferente experiencia que deja como resultado la compra del producto, donde finalmente la marca logra su objetivo.

Es decir, consiste en la denominada "tienda espectáculo", y pasa por la concepción del mo- 
biliario específico, la decoración, la información, etc., con el objetivo de dar un aspecto seductor al lineal y a la tienda, para promover la imagen del propio distribuidor.

Teniendo en cuenta que el consumidor llega a conocer los productos a través de todos los sentidos (un $55 \%$ a través de los ojos, un $18 \%$ a través del oído, un $12 \%$ del olfato, un $10 \%$ del tacto y un $5 \%$ del gusto), se puede utilizar esta información para animar el punto de venta.

Es necesario crear un ambiente agradable en el comercio, ya que ayuda a la venta visual presentando una tienda bien decorada y bien iluminada. Se debe estudiar el nivel de claridad conveniente en función de los productos, de su presentación y de la decoración en general.

Es un hecho comprobado que los consumidores acuden más a las zonas más iluminadas, pues una claridad elevada aumenta la rapidez de la percepción visual, permitiendo a los compradores reaccionar más rápidamente y circular con mayor comodidad.

La organización de acciones promocionales es otro de los aspectos a tener en cuenta. Algunos elementos publicitarios estratégicamente dispuestos permitirán llamar la atención del público sobre los stands de demostración, avisar a la clientela cada vez que se produzca una demostración y anunciar las ventajas promocionales que se ofrezcan en la tienda.

\section{Según la Situación del Cliente}

\section{a. El cliente Shopper:}

También llamado Cliente Oportunista es aquel que necesita una serie de argumentos para acudir a un establecimiento y no a otro. Quiere saber dónde hay que comprar y no qué comprar. Le preocupan aspectos tales como la facilidad de acceso, el aparcamiento, la imagen del establecimiento y los precios en general.
Al cliente shopper se le aplicará un marketing de entrada para conseguir captar nuevos clientes y fidelizar a los captados anteriormente. En resumen, el cliente shopper es el que, fuera del establecimiento, elige dónde quiere o le conviene más comprar. El cliente buyer es el que, dentro del establecimiento, decide qué va a comprar.

\section{b- El cliente Buyer:}

Conocido como Cliente Comprador: es el cliente dentro del establecimiento, cuando ya está dentro del punto de venta; basará su decisión de compra en los precios, la calidad de los productos, las distintas ofertas, las promociones, la gama de productos y el trato recibido.

Al cliente buyer se le aplicará un marketing de salida con el fin de que adquiriera no sólo los productos que buscaba, sino aquellos que no tenía previsto comprar.

Esta distinción teórica del cliente shopper y el cliente buyer sirve para que las empresas no descuiden ni los factores externos que hacen que un cliente entre en el local, ni los factores internos que hacen que el cliente compre determinados artículos.

La tendencia actual es la comodidad, y muchos comercios excelentes por dentro descuidan lo referente a la atracción requerida en los aspectos shopper, tales como la señalización adecuada para que el cliente sepa cómo ir al local o la numeración de las plazas de aparcamiento, por citar algunos.

Por lo contrario, otros comercios han cuidado bastante los aspectos shopper, pero la cortesía y atención al cliente dejan mucho que desear.

En definitiva, deben cuidarse ambos aspectos para que el establecimiento vaya bien (Cubillos \& Laverde, 2009).

\section{Caso Tunning Exports.}


Después de haber analizado a fondo el caso de la empresa de la referencia, se entrevistó al Sr. Juan José Sarué Montalvo, propietario de la empresa, quien manifestó su interés sobre manera de este tema tan vital para generar productividad y por ende rentabilidad.

Es de anotar que la entrevista estructurada se realizó en la sede de la empresa, fundada en el año 2008 y que está situada en la Cra. 46\# 82-67 de la ciudad de Barranquilla.

Bueno, esta empresa se inició en el año 2008 por mi persona y mi socio Nicolás Rúgeles, que simplemente vimos la necesidad que había de importar accesorios de carros y que no había aquí en el país una actual oferta que pudiera traer accesorios que no existían de pronto en el mercado. Entonces, simplemente se pedían por fuera y se traían a los clientes que tenían la demanda o necesidad de adquirirlos y no encontraban una forma de poder comprarlos en el país. Así empezó, online también, por internet a través de mercado libre. Iniciando con un $50 \%$ del pedido inicialmente, y el resto apenas llegara. Para después ir viendo cuales eran los artículos de más demanda y simplemente tenerlos siempre en inventario (Tunningimports.com, 2018; Ver anexo 1).

Desde el punto de vista de merchandising fue poco lo que realizaron los socios, la inicio de la misma. Pero ya tenían una visión digital para comercializar los productos. Desde la óptica del merchandising físico y de material publicitario, solo se ve en el almacén de manera sencilla, algunos avisos o sugerencias para los clientes, que en su mayoría son fieles y leales. Sin embrago hacen falta estrategias claras para este fin.

No obstante, su sistema de marketing digital es bueno, sobre todo desde su página web. En donde se observa con suma atención sus promociones en Facebook, Instagram, Twitter y WhatsApp. Es una página interactiva y actualizada, aunque no tan interactiva como podría tener la competencia.
Con base en la investigación realizada, y teniendo como base a esta empresa, se pueden inferir para este tipo de empresas los siguientes direccionamientos desde el merchandising:

- Merchandising permanente. Las empresas de este tipo suelen hacer merchandising permanente, debido a que sus ciclos de promoción son largos. Es por ello que teniendo como base esta investigación, se decidio que estas empresas hacen merchandising permanente para:

- Captación de clientes potenciales, ya que, en la mayoría de los casos, los leales y fieles solo necesitan estrategias de mantenimiento.

- Generar vínculos establecimiento - cliente (el cual reconoce y se identifica con el almacén). Para este caso, se deben hacer promociones $\mathrm{y} / \mathrm{o}$ estrategias que permitan la estadía de fidelidad del cliente.

- Ambientación acorde con la estratificación de clientes. (se recuerda que son repuestos y lujos para automóviles de gama alta).

- Él establecimiento, desde su fachada hasta el final de los talleres y o patios, la distribución del espacio, la gestión del área expositiva (tipo de surtido, tipos presentación, criterios colocación.

Merchandising promocional, se aplica con el lanzamiento de un nuevo producto, y/o servicio. No se puede olvidar, que este tipo de empresas, también existe el servicio de taller para estos automóviles, tal y como se aprecia en la ilustración 5. 


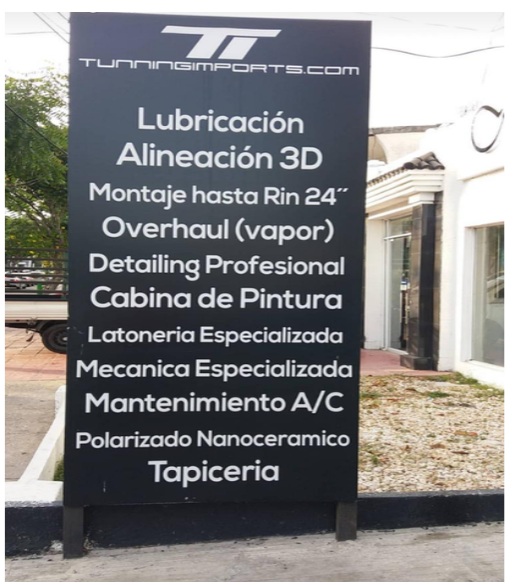

Ilustración 5: Merchandising promocional

Fuente: Imagen tomada con fines académicos de Tunning sports

- Mayor agresividad comercial. Con esto se da a entender porque somos o queremos ser líderes en el mercado. Un ejemplo de ello es, inclusive es la relación precio/ servicio, a pesar de que el establecimiento es de estrato alto. Se aprecia en la ilustración 6, este tipo de estrategia:

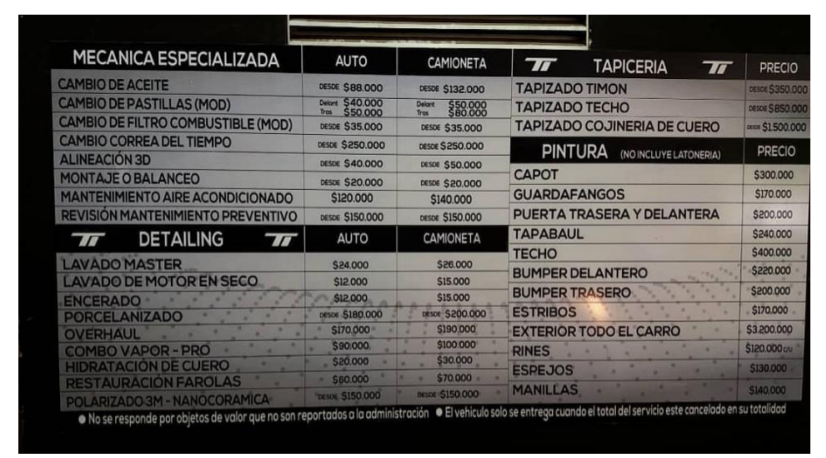

Ilustración 6: Merchandising promocional

Fuente: Imagen tomada con fines académicos de Tunning sports

- Cartelería de señalización.

- Merchandising Estacional: Es el que se realiza en virtud de la relación de los productos con la época del año. Para el caso de estas empresas, no aplica, ya que la estacionalidad se da todo el año.

\section{Según su Naturaleza}

- a. Merchandising de Presentación: Para este tipo de negocios es de suma importancia este tipo de merchandising, ya genera un marketing de atracción al cliente que llega al establecimiento.

Los productos y el establecimiento son organizados de tal forma que comprar resulte fácil tanto como para el cliente, además de rentable para el comerciante. También se le denomina como merchandising visual, donde su filosofía es que todo lo que se ve, se vende; y viceversa. Este tipo de merchandising para estos establecimientos provoca que el consumidor compre de manera impulsiva o algún producto que no haya previsto anteriormente. La base de este tipo es que el lugar sea agradable, todo esté distribuido y en su posición adecuada y un surtido adecuado que satisfaga las necesidades de los consumidores. Esto teniendo en cuenta que las ventas son de mostrador.

\section{Macro y Micro Visual merchandising.}

- Cabe diferenciar el macro espacio de un establecimiento con el micro espacio de una zona en concreto del mismo. Ver llustración 7

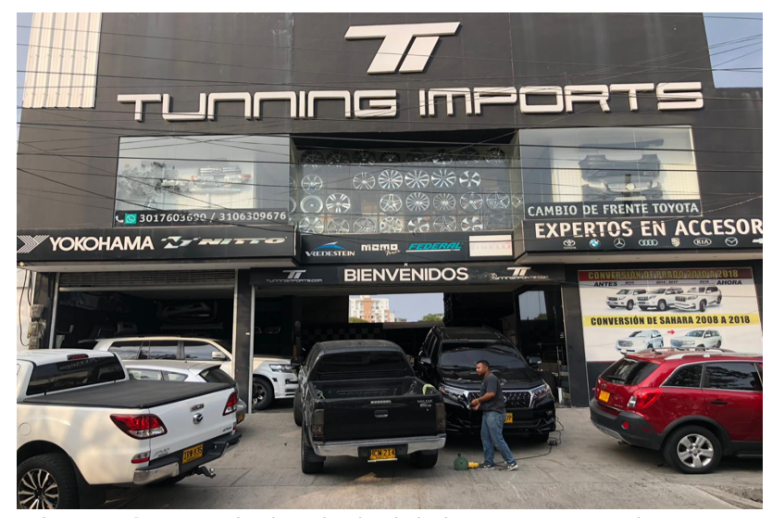

Ilustración 7: Fachada principal de la empresa Tunning Exports. Fuente: Imagen tomada con fines académicos de Tunning Imports.

\section{Merchandising de Gestión.}

Consiste, como su nombre indica, en gestionar el espacio para obtener el máximo rendimiento posible del lineal (espacio destinado por el establecimiento a la exposición y venta de los productos). 


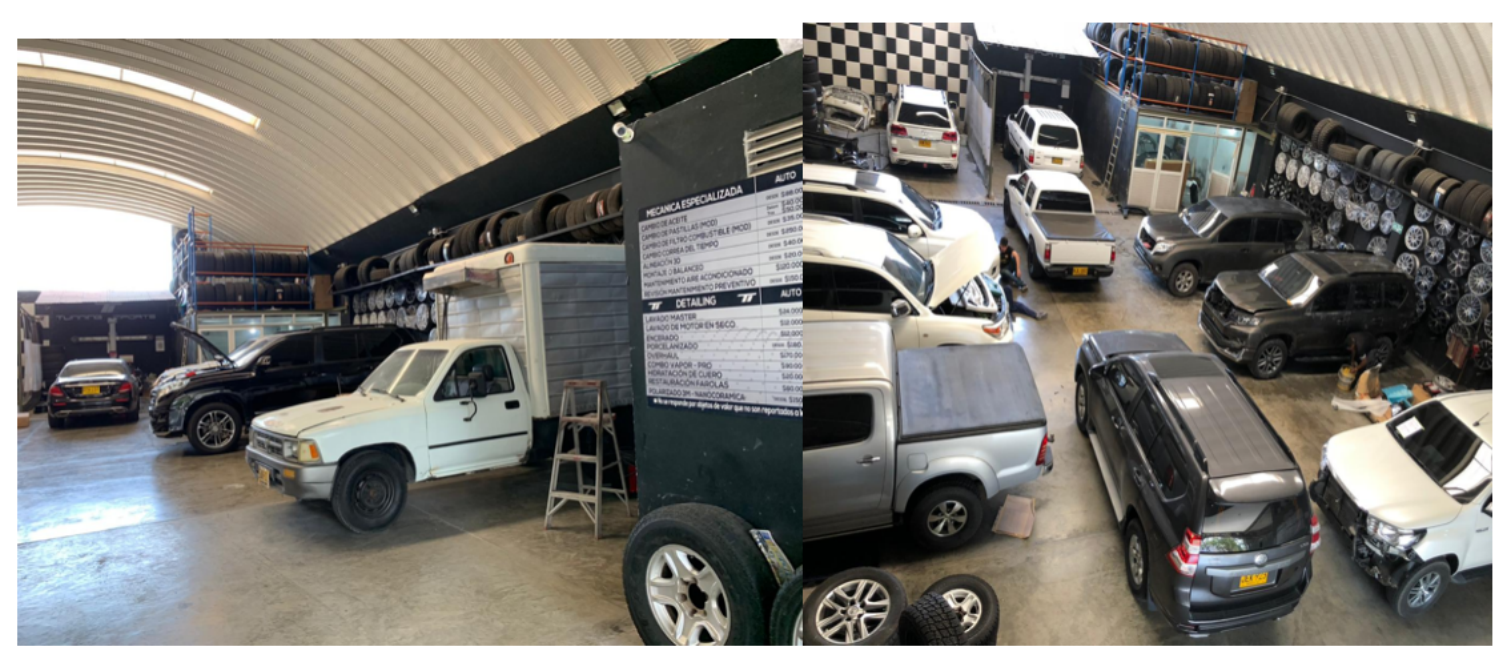

Ilustración 8: Aspectos del taller de Servicios.

Fuente: Imagen tomada con fines académicos de Tunning Imports.

El merchandising de gestión se basa en la gestión del espacio, consistente en una mejor optimización del espacio, tal y como se aprecia en la imagen. Los vehículos son situados en los laterales del taller para darle paso por el centro a los vehículos que salgan y entren en él. El establecimiento tiene un gran poder de recomendación en todos los servicios. Aunado a la publicidad, y a la exhibición de productos de manera física, gestionan un ambiente de gran calidez para el marketing de atracción para los clientes. Al consumidor le gusta ver estas marcas expuestas en el punto de venta, ya que identifican ese lugar como próximo y dotan de prestigio al establecimiento.

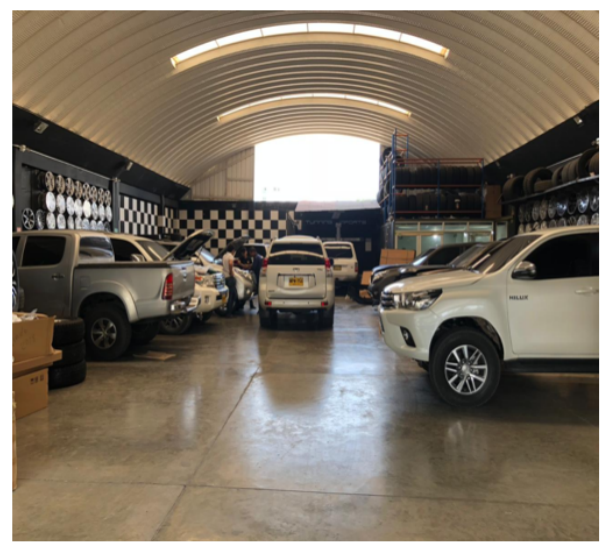

Ilustración 9: Fachada Central de Servicios Fuente: Imagen tomada con fines académicos de Tunning Imports
Para ello, es necesario que el lugar destinado a la venta disponga del mayor espacio posible. La gestión de este espacio de exposición busca su optimización económica.

\section{Merchandising de Seducción o animación}

El marketing sensorial, también llamado de seducción, está siendo implementado por numerosos negocios, que logran que sus usuarios y consumidores reciban impulsos de forma inconsciente a través de la evocación de sensaciones y la recepción de percepciones agradables.

El merchandising de seducción se basa en la tienda espectáculo, centrada en la estimulación de los cinco sentidos del consumidor, por lo que sus claves deben ser oler (aroma), saborear, sentir, mirar y escuchar. Esta táctica suele funcionar de manera más efectiva cuando se trata de un producto que emocionalmente vinculado al cliente.

Este tipo de estrategia destruye los clásicos estereotipos comerciales que rezan que el consumidor se guía en exclusiva por factores de precio o la calidad al momento de la compra. Aunque estos sean factores importantes, no son decisivos, y muchas de las compras por impulso o complementarias, incluso de productos de primera necesidad, 
también responden a estímulos generados por el merchandising de seducción.

Por tanto, en esta clase de merchandising priman las emociones, las percepciones y las sensaciones sobre el raciocinio. No se utiliza de forma aislada, sino como complemento a las tradicionales técnicas de marketing y comunicación que pretende estimular la parte irracional del ser humano. Su objetivo es crear emociones a través de los cinco sentidos de una persona para establecer un vínculo personal entre un establecimiento y sus clientes.

Es una táctica que permite proporcionar en el punto de venta una experiencia mucho más completa que estimule a las personas a sentir, soñar, relajarse, disfrutar, sorprenderse, reír y experimentar emociones.
Uno de los motivos del poder sugestivo que tiene esta estrategia es que, por ejemplo, en el caso del sistema olfativo, la información llega al cerebro a través del sistema límbico y el hipotálamo, regiones cerebrales responsables de las emociones, los sentimientos, los instintos e impulsos, la memoria y la liberación de hormonas. (neuromarketing).

Los olores no pasan por el córtex, que se trata de la zona racional, por lo que tienen un alto poder sugestivo. Dentro del merchandising de seducción también tiene cabida el auditivo.

Para el caso de la empresa en mención, se hace uso de lo visual, ya que a través de la web y del marketing exterior, éstos aprovechan la espectacularidad de los lujos y repuestos para este tipo de automóviles. A través de la web, se observa, por ejemplo la interactividad visual de los banners de la misma.
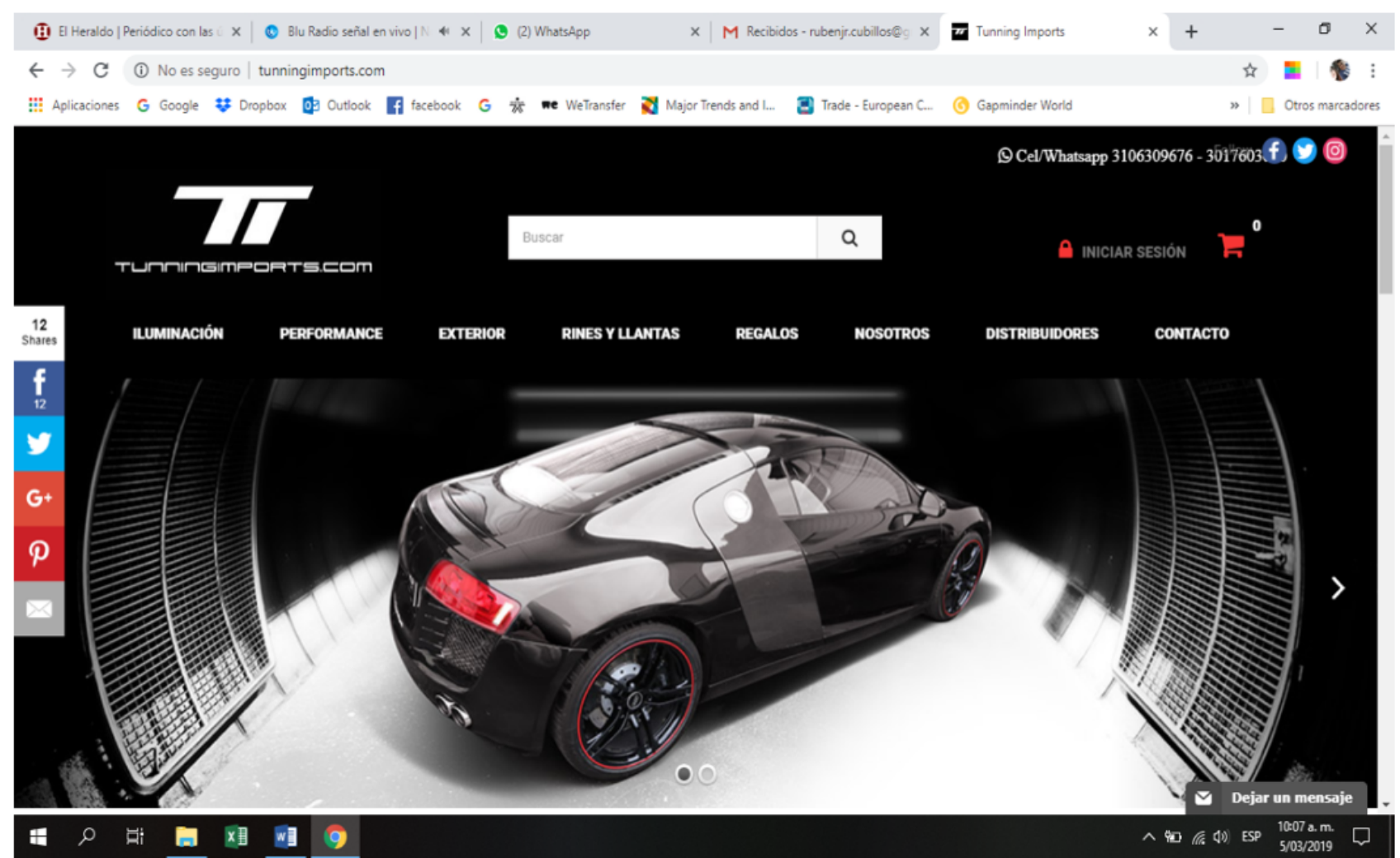

Ilustración 10: Home page de Tunning Imports

Fuente: Imagen tomada con fines académicos de Tunning Imports 


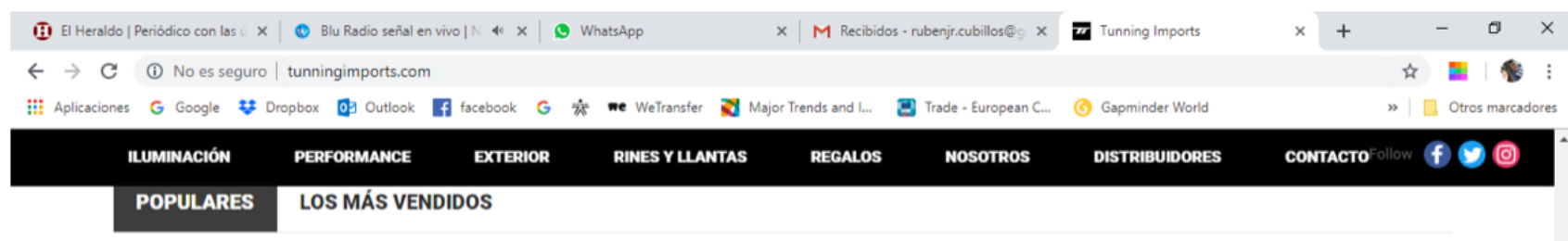

Ilustración 11: Link de los artículos más populares

Fuente: Imagen tomada con fines académicos de Tunning Imports

\section{Según la Situación del Cliente}

\section{a. El cliente Shopper:}

Este tipo de establecimiento cuenta con este cliente, el cual le preocupan aspectos tales como llegar fácilmente, el parqueadero, la imagen del establecimiento y los precios en general. Acorde con la encuesta (ver anexo 1) realizada a través de un sondeo, se escogieron al azar 14 clientes de tipo shopper y de tipo buyer. Al cliente shopper se le aplicará un marketing de entrada para conseguir captar nuevos clientes y fidelizar a los captados anteriormente. En resumen, el cliente shopper es el que, fuera del establecimiento, elige dónde quiere o le conviene más comprar.

\section{b. El cliente Buyer:}

Conocido como Cliente Comprador: es el cliente dentro del establecimiento, cuando ya está dentro del punto de venta; basará su decisión de compra en los precios, la calidad de los productos, las distintas ofertas, las promociones, la gama de productos y el trato recibido.

Al cliente buyer se le aplicará un marketing de salida con el fin de que adquiriera no sólo los productos que buscaba, sino aquellos que no tenía previsto comprar.

Esta distinción teórica del cliente shopper y el cliente buyer sirve para que las empresas no descuiden ni los factores externos que hacen que un cliente entre en el local, ni los factores internos que hacen que el cliente compre determinados artículos.

La tendencia actual es la comodidad, y muchos comercios excelentes por dentro descuidan lo referente a la atracción requerida en los aspectos shopper, tales como la señalización adecuada para que el cliente sepa cómo ir al local o la numeración de las plazas de aparcamiento, por citar algunos.

Por lo contrario, otros comercios han cuidado bastante los aspectos shopper, pero la cortesía y 
atención al cliente dejan mucho que desear.

En definitiva, deben cuidarse ambos aspectos para que el establecimiento vaya bien. En Conclusión , la diferencia del cliente shopperes que se enfoca en donde hay que comprar y no en el que hay que comprar, y se centra cuando está en el establecimiento sobre el fácil acceso de compra, el establecimiento y su imagen y los precios generales; y el cliente buyer es el que se enfoca cuando está dentro del establecimiento, se percata de los precios, calidad del producto, las ofertas, promociones, y la gama de productos y del trato recibido. En resumen, el cliente shopper es el que, fuera del establecimiento, elige dónde quiere o le conviene más comprar. El cliente buyeres el que, dentro del establecimiento, decide qué va a comprar.

\section{Resultados de la investigación primaria.}

Teniendo en cuenta que se encuestaron a los clientes tanto buyer como shopper, la investigación arrojó con respecto al merchandising, resultados interesantes que se explican a continuación:

El $43 \%$ de los encuestados conocen la empresa a través de la pagina web y el $36 \%$ pasando por el lugar y por la fachada. Esto indica la favorabilidad de la empresa en sus estrategias de merchandising desde lo digital. Así, como de su merchandising ex- terior debido a su excelente ubicación, facto principal para atraer clientes.

La atracción hacia la empresa, lo que se conoce como marketing de atracción en el merchandising, son sus productos y/o servicios en un $57 \%$, lo que constituye una excelente labor en el área de exhibición, a pesar de ser productos de alta gama y en algunos casos de gran tamaño.

En cuanto a la satisfacción del cliente con respecto a la empresa, la encuesta arrojó que, el $71 \%$ de los encuestados están plenamente satisfechos con los productos que allí se ofrecen. Esto aunado a su exhibición, que se expuso en el comentario anterior, corrobora la importancia, desde lo visual para la exhibición.

Por otro lado, el aspecto físico, tanto interior como exterior es muy importante, ya que representa la comodidad del cliente. Es así como el $86 \%$ de los encuestados resaltaron los espacios de la empresa. Esto muestra lo estratégico y táctico que es para la consecución de los objetivos gerenciales. Tal y como lo resalta la teoría en su variable que tiene relación con la arquitectura de la tienda.

Otras variables importantes, que resaltaron los clientes son las siguientes: en un $36 \%$, la importancia

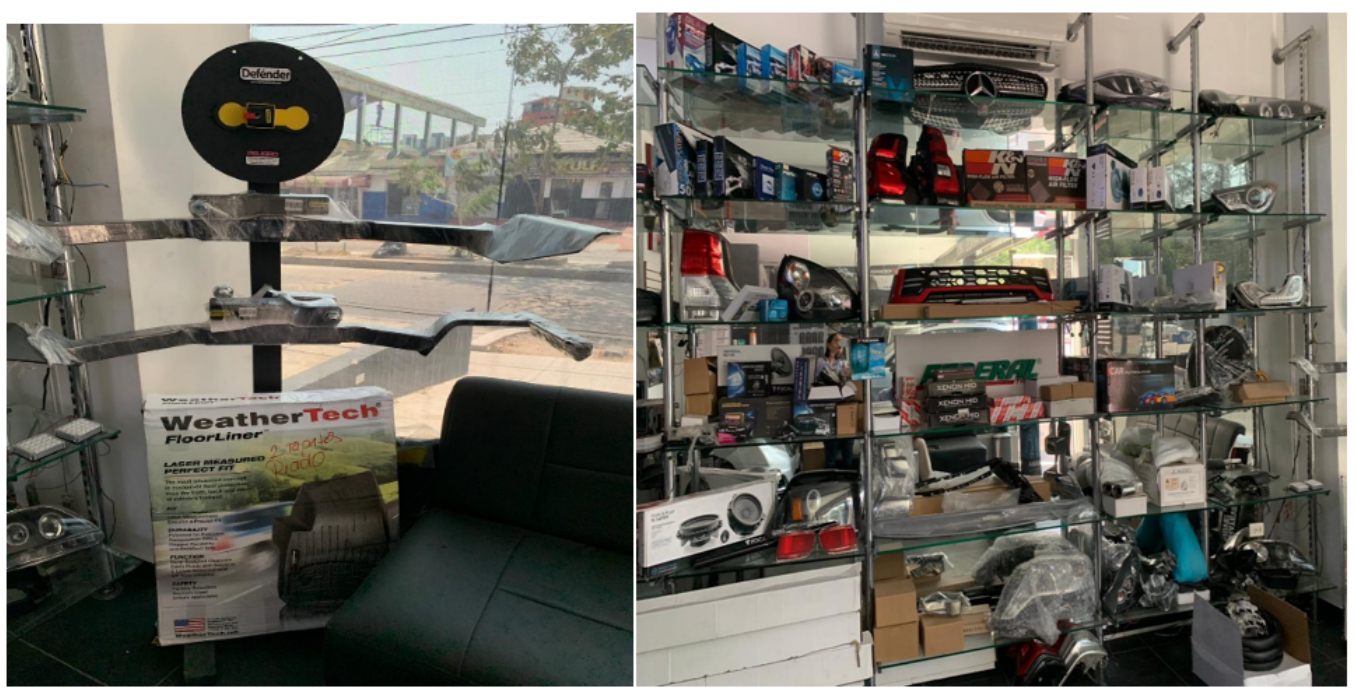

Ilustración 12: Exhibición de productos

Fuente: Imagen tomada con fines académicos de Tunning Imports 
de la limpieza y el orden de la empresa, así mismo, los espacios y la comodidad que representa para los clientes, sobre todo, para los tiempos de espera.

\section{CONCLUSIONES}

Como conclusión, después de haber abordado todos los aspectos más relevantes para este tipo de establecimientos, el merchandising aparece como un factor que genera productividad, $y$, por ende, rentabilidad. Para tener en cuenta, este debe gestionar entre otros los siguientes aspectos:

- Superficie disponible requerida para este fin, es decir, el espacio suficiente para el tráfico tanto de vehículos como personas.

- Lugar atractivo: para el marketing de seducción es importante el impacto que genere al comprador un lugar de impacto, sobre todo para este tipo de clientes de gama alta para sus productos y servicios.

- Facilidades para la prestación del servicio. Para este punto es importante resaltar las entregas oportunas, tiempos de espera, promesa cumplidas, entre otros.

- Productos a la vista de los clientes. Que no opere con un vendedor, sino que este pueda, a través de su propio impulso, observar los productos y hacerse una percepción de los servicios.

- Libre acceso del producto a los clientes: El mostrador en este tipo de negocios, no es estratégico, el libre acceso a la exhibición para los clientes, hace que el merchandising sea más atractivo.

- Realización de merchandising permanente, promocional y estacional. Para este tipo de establecimiento deben operarlos tres, ya que para este tipo de segmento es crucial el visual marketing en todas sus modalidades.
- Merchandising de seducción principalmente, ya que para estos tipos de productos de alta gama es necesario seducir al segmento e impactarlo sobremanera.

- Estrategias para clientes shopper y buyer, ya que en este tipo de establecimientos se dan los dos, teniendo en cuenta esto, las estrategias difieren para cada uno. 


\section{$\sim$ Referencias bibliográficas}

Cubillos, R. \& Laverde, J. (2009). La fantasía del merchandising.

Hasty, R. \& Reardon, J. (1998). Gerencia de ventas al detal. Bogotá, Colombia: McGraw Hill.

Laverde, M. (2015). Marketing para droguerías. Editorial Santa bárbara.

Lerma, A. (2003). El merchandising. Thomson Business Journal.

Tunningimports.com (26 de febrero de 2018). http:// tunningimports.com/content/4-nosotros 